\title{
ОЦЕНКА ВЛИЯНИЯ ГЕОДИНАМИЧЕСКИХ ЗОН, ПЕРЕСЕКАЮЩИХ МАГИСТРАЛЬНЫЕ ГАЗОПРОВОДЫ, НА ИХ НАПРЯЖЕННО-ДЕФОРМИРОВАННОЕ СОСТОЯНИЕ
}

\author{
Аскаров Роберт Марагимович', \\ askarov1943@mail.ru \\ Валеев Анвар Рашитович', \\ anv-v@yandex.ru \\ Исламов Ильдар Магзумович², \\ mildarislamov@mail.ru

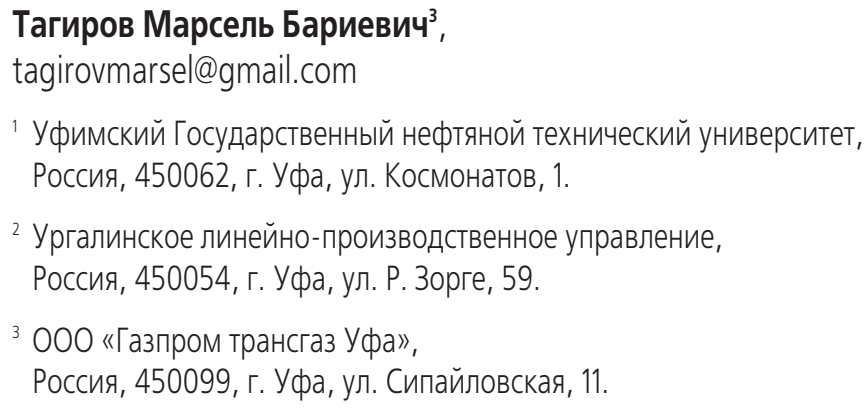

Актуальность. Одной из причин, способной разрушать газопроводы, являются геодинамические воздействия, так как по мере длительной эксплуатации газопроводов, расположенных, например, на тектонических разломах, происходит накопление повреждающего фактора, связанного с изгибными напряжениями. Анализ фактического положения газопроводов по данным внутритрубного технического диагностирования показал наличие потенциально-опасных участков, на которых кривизна не соответствует проектной, а значит изгибные напряжения также могут значительно отличаться по абсолютной величине в большую сторону от проектных. Следовательно, необходимо своевременно выявлять такие потенциально-опасные участки, и в зависимости от уровня фактических напряжений проводить восстановительные мероприятия.

Цель: провести оценку влияния геодинамических зон, пересекающих магистральные газопроводы на их напряженно-деформированное состояние с использованием данных внутритрубного технического диагностирования, в т. ч. с учетом их длительной эксплуатации.

Объект: участок четырехниточного коридора магистрального газопровода, расположенного на территории западного Урала, где по данным Российской Академии Наук в настоящее время наблюдаются надвиги.

Методы: аналитические методы исследования напряженно-деформированного состояния магистральных газопроводов по данным о пространственном положении его оси, полученных методами общепринятой технологии и данными внутритрубной диагностики

Результаты. Проведен анализ основных этапов общепринятой технологии выявления геодинамических зон и определения его основных параметров, оценки напряженно-деформированного состояния. Показана возможность выявления потенциальноопасных участков магистральных газопроводов на пересечениях с геодинамическими зонами, по данным внутритрубной диагностики. Установлено, что с увеличением сроков эксплуатации магистральных газопроводов возрастает количество потенциально-опасных участков, на которых радиус кривизны не соответствует требованиям нормативной документации по минимально допустимому, равному 1000 D. По результатам обработки данных внутритрубной диагностики приведены примеры определения величин изгибных напряжений, превышающих нормативные значения. В связи с невозможностью полной переукладки магистральных газопроводов, предлагается привести нормативные требования к уровню напряжений. Кроме того, показана возможность выявления активных геодинамических зон и их параметров, по данным внутритрубной диагностики.

\section{Ключевые слова:}

Магистральный газопровод, геодинамические зоны, напряженно-деформированное состояние, потенциально-опасные участки, внутритрубное техническое диагностирование.

\section{Введение}

При проектировании газопроводов в большинстве случаев выбор трасс ограничен; они нередко пересекают геодинамические зоны (ГДЗ) [1-4]. К таким зонам относятся: движения земных блоков, надвиги (горные удары), карсты, тектонические разломы, разломы другого характера и т. п.

По данным Российской Академии наук (РАН), на западном Урале были обнаружены и прослежены надвиги, вызвавшие образование зон деформа- ций $[5,6]$ на 400 км. При надвигах образуется отрицательный рельеф местности, т. е. со стороны надвигов образуется более крутой склон по сравнению с более пологим на другой стороне. Предполагается, что надвиги, воздействуя на магистральный газопровод (МГ), приводят его в движение, изменяя его напряженно-деформированное состояние (НДС).

В настоящее время установлено, что причиной самой масштабной аварии XX в. в трубопроводном 
транспорте (продуктопровод в Республике Башкортостан) [7] является геодинамический фактор [5], и поэтому особую важность приобретает оценка результатов воздействия активных ГДЗ на МГ.

\section{Выявление геодинамических зон}

Основные этапы выявления ГДЗ покажем на примере четырехниточного коридора МГ, проходящего по западному Уралу с севера на юг. Технология выявления ГДЗ и их параметров, где выделяются два этапа:

- анализ внешних признаков с использованием аэрокосмических технологий;

- анализ таких данных геолого-геофизических технологий, как параметры ГДЗ, размеры, направление и скорость перемещения блоков.
В качестве примера на рис. 1 приведена карта с результатами структурно-тектонического дешифрирования материалов космической и аэрофотосъемки (участки ГДЗ № 0-12) в месте расположения МГ.

На рис. 1 показано, что участок МГ пересекает ГДЗ линейной трещиноватости (участки № 1,3 , $5-7,10,12)$ и изометрической трещиноватости (№ $2,4,9)$. Согласно рис. 1 , зона активного вертикального движения земли со скоростью до $1,7 \ldots 1,8$ мм/год располагается в районе ГДЗ № 12. Результаты геолого-геофизических исследований ГДЗ № 12 [8] приведены на рис. 2.

На рис. $2 \mathrm{a}-\mathrm{b}-\mathrm{c}$ - ось МГ, маркеры $\mathrm{O}_{1}$ и $\mathrm{O}_{2}$ обозначают места шурфовок. Скорость поднятия земли в ГДЗ достигает 1,8 мм/год. Примерно с той же
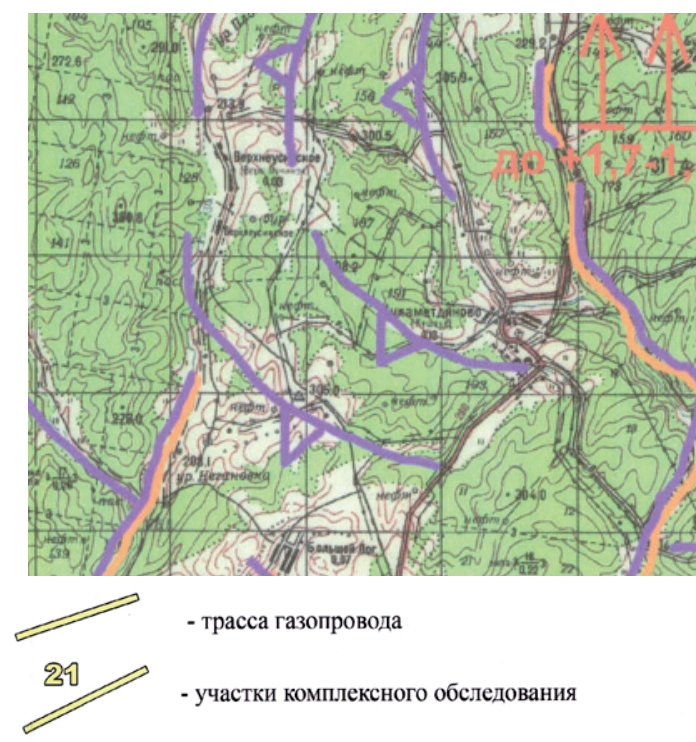

- трасса газопровода
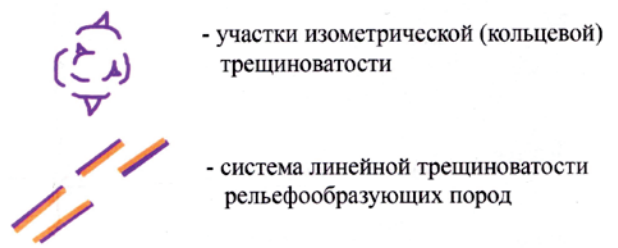

| 1 - $\begin{gathered}\text { - линейная зона дробления рельефообразующих } \\ \text { пород с правосторонним сдвигом }\end{gathered}$
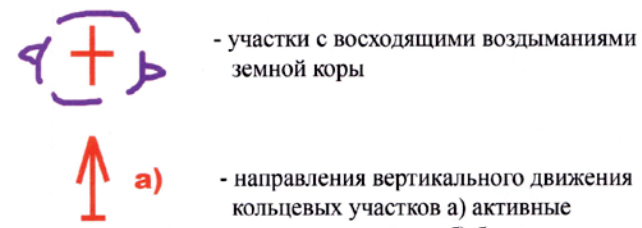

б) более активные

1 (16)

от 1,7 до 1,8 мм/г - скорости воздымания кольцевых участков

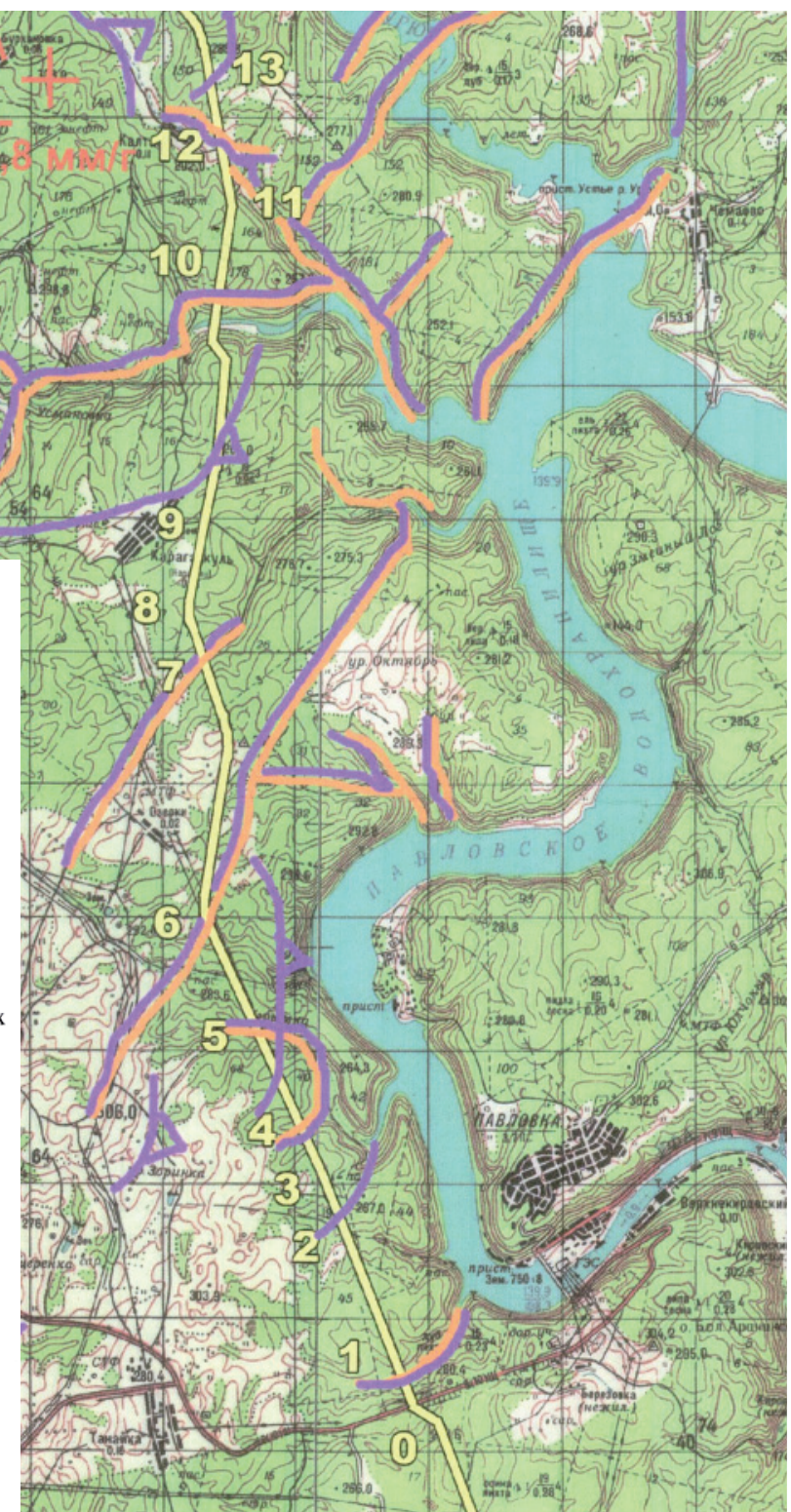

Pис. 1. Результаты структурно-тектонического дешифрирования материалов космической и аэрофотосъемки (участки № 0-12)

Fig. 1. Results of structural tectonic interpretation of materials for space and aerial photography (sections 0-12) 


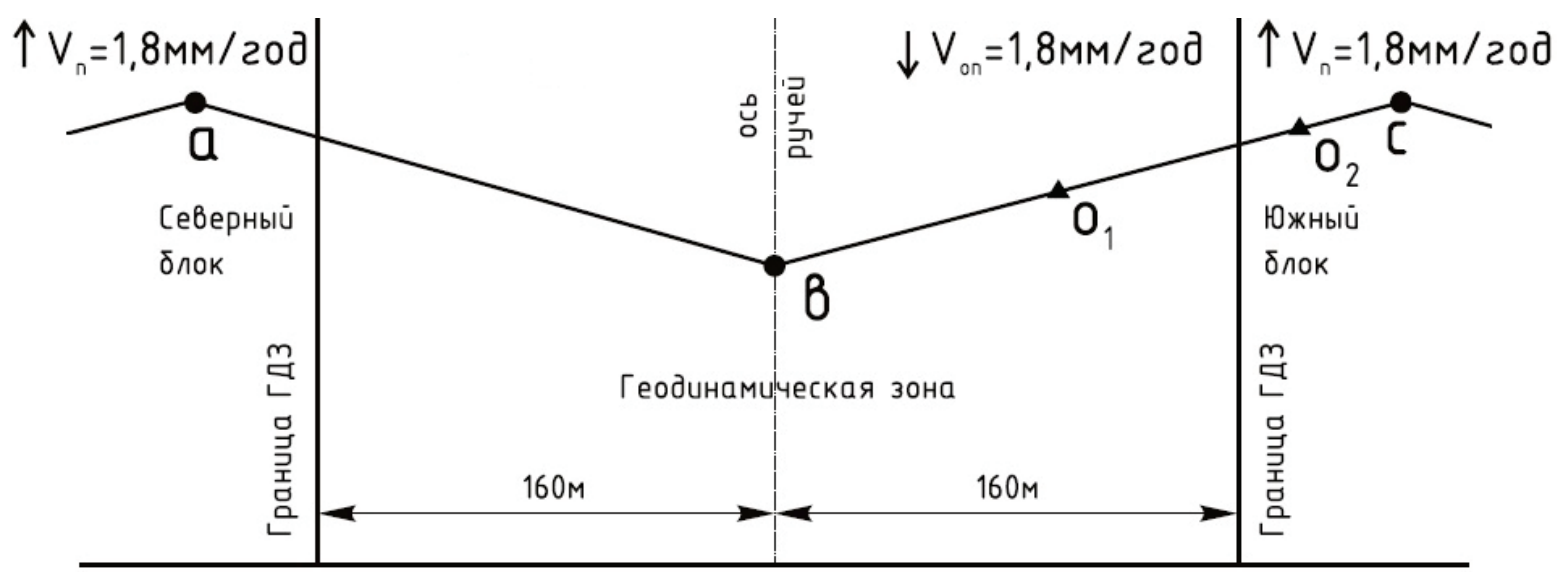

Puc. 2. Схема параметров ГДЗ № 12

Fig. 2. Diagram of the parameters of geodynamic zone № 12

скоростью опускается зона разлома, имеющего выраженные границы и глубокое простирание. По положению оси газопровода очевидно направление воздействия ГДЗ. В центральной части выпуклого участка движение грунта происходит вниз, а по краям - вверх.

Вывод о степени воздействия ГДЗ на НДС МГ может быть сделан после измерения фактических напряжений в шурфах. Одним из методов измерения абсолютных значений напряжений в деталях и конструкциях является ультразвуковой, получивший широкое распространение как в отечественной, так и зарубежной практике. К системе, в которой реализован этот метод, относится измерительно-вычислительный комплекс «Астрон», в основу работы которого положен спектральный импульсный метод акустической структурометрии. Способ измерения напряжений в комплексе «Астрон», как и у большинства способов измерения НДС, контактный. Это требует удобного подхода к стенке трубы, что достаточно сложно реализовать, например на подводных переходах, в пойменной части и т. п.

\section{Измерения напряженно-деформированного состояния в шурфах на пересечении с геодинамической зоной № 12}

В табл. 1 приведены значения измеренных комплексом «Астрон» продольных напряжений в шурфах № 12/1, 12/2 [9].

Таблица 1. Значения измеренных продольных напряжений Table 1. Values of measured longitudinal stresses

\begin{tabular}{|c|c|c|c|c|c|c|c|}
\hline \multirow{2}{*}{$\begin{array}{c}\text { Hoмep шурфа } \\
\text { Pit number }\end{array}$} & \multicolumn{7}{|c|}{$\begin{array}{c}\text { Продольные напряжения, МПа - } \\
\text { в часовых координатах }\end{array}$} \\
\cline { 2 - 8 } & $7^{\frac{30}{0}}$ & $9^{00}$ & $10^{30}$ & $12^{00}$ & $1^{30}$ & $3^{00}$ & $4^{30}$ \\
\hline $12 / 1$ & 80 & 160 & 220 & 200 & 120 & -70 & -190 \\
\hline $12 / 2$ & -150 & 50 & 110 & 120 & 170 & 20 & -140 \\
\hline
\end{tabular}

Учитывая, что радиусу кривизны, равному $1000 D$, соответствуют по правилам [10] напряже- ния величиной около 100 МПа, в обоих измеренных сечениях при максимальных напряжениях, равных 220 и $170 \mathrm{MПа,} \mathrm{радиусы} \mathrm{изгиба} \mathrm{составят,}$ соответственно, 450 и $590 \mathrm{D}$, что не соответствует нормативным значениям правил [11].

Анализ положения газопровода в шурфах показал, что в обоих шурфах трубопровод расположен выпуклостью вверх, что подтверждает перемещение южного блока границы ГДЗ вверх. А так как максимальные растягивающие напряжения в шурфах не совпадают по часам, то можно говорить и о наличии отклонения газопровода от проектного положения в плане.

Согласно полученным данным, продольные напряжения соответствуют нормативу [10]. Тем не менее это не означает, что фактическая картина НДС благополучная, скорее это свидетельствует 0 несовершенстве общепринятых вероятностных методов оценки потенциально-опасных участков (ПОУ) на пересечениях с ГДЗ, основанных на ряде допущений. Перечислим некоторые из них: достоверность выявления собственно ГДЗ с применением аэрокосмических технологий, определения его параметров: протяженности, направления и скорости движения блоков геолого-геофизическими методами; перемещения трубопровода в полном соответствии с перемещением блоков; места выбора шурфовки под измерение НДС; точности измерения НДС и т. д.

\section{Определение потенциально-опасных участков а пересечениях с геодинамическими зонами по данным внутритрубного технического диагностирования}

За последнее время ВТД газопроводов [12] развивается в направлении позиционирования пространственного положения газопровода, к настоящему времени технология определения профиля и радиуса изгиба [13-15] газопровода позволяет уже с достаточной для инженерных расчетов точностью оценивать напряжения от изгиба. Метод позволяет надежно отображать линейно-высотное 


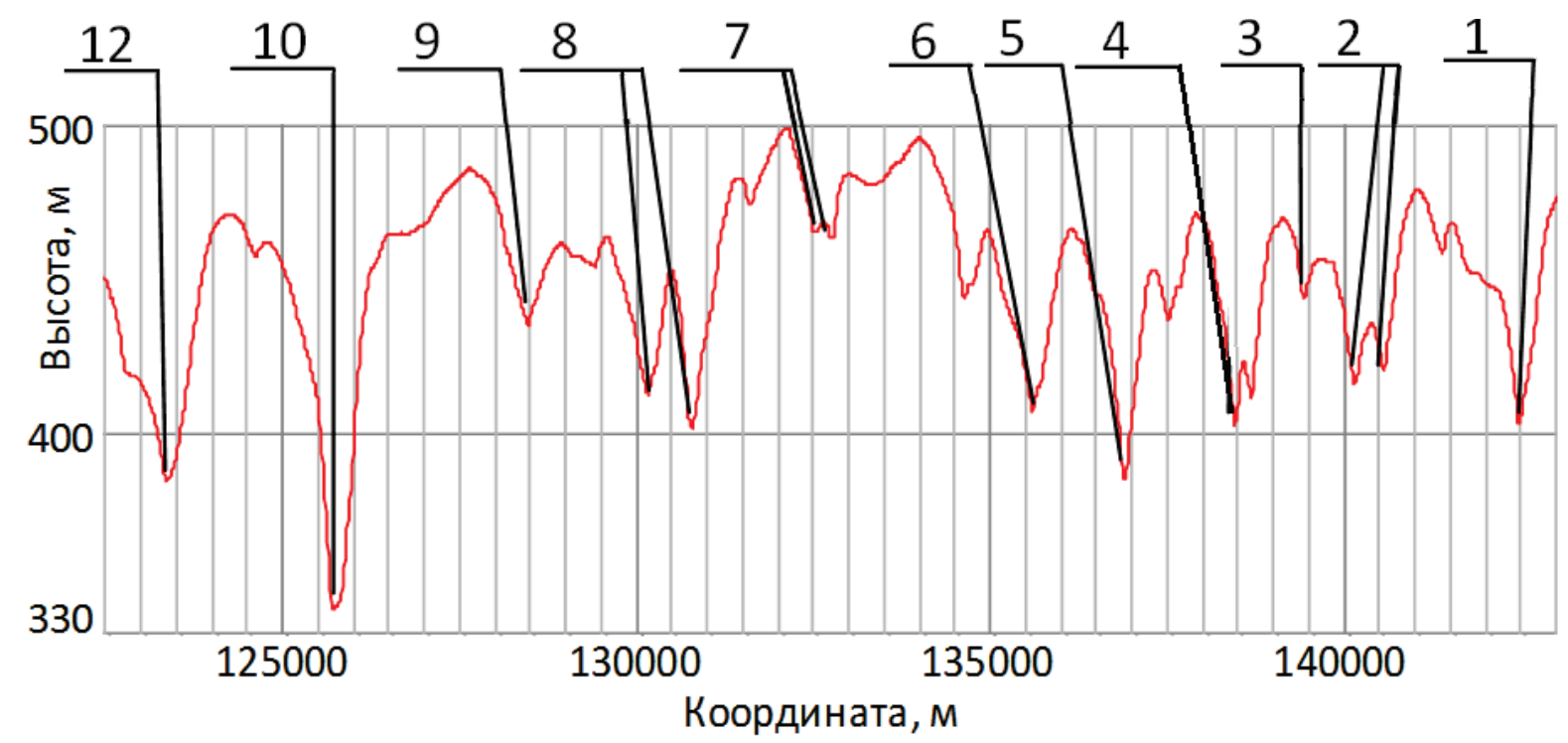

Рис. 3. Профиль участка на пересечениях с ГдЗ

Fig. 3. Pipeline profile at the intersections with geodynamic zones

положение участка газопровода и измерять радиус изгиба от $4000 \mathrm{m.}$

Если схему на рис. 1 можно отнести к плану участка МГ, то применительно к трубопроводному транспорту значительно большей информативностью обладает профиль участка МГ. На рис. 3 приводится профиль участка МГ, пересекающего ГДЗ № 1-12, построенный по данным ВТД [16].

Анализ профиля участка, изображенного на рис. 3 , показывает, что:

- надвиги ГДЗ направлением справа налево (ГДЗ

№ $1,2,4,6,8,10,12)$ вполне различимы;

- ГДЗ № 3, 7, 9 можно рассматривать не столько как собственно ГДЗ, а как следствие деления крупных блоков ГДЗ № 2, 6, 8 на более мелкие [5].

Из вышеизложенного следует, что на профиле участка в виде ГДЗ отражены сколько-нибудь значимые ПОУ, в том числе и складки местности, и, следовательно, нет необходимости проводить аэрокосмические исследования.

На рис. 4 приведен график радиусов изгиба МГ на пересечении с ГДЗ № 12, построенный по данным ВТД 2015 г. [13, 16]. Синим цветом выделены участки с отводами холодного гнутья $(О \mathrm{XГ})$, зеленым - сечение с минимальным радиусом изгиба в упругой зоне. Горизонтальные прямые выделяют следующие радиусы изгиба:

- $1000 D$ и более допустимы, согласно правилам $[10,11]$

- $500 D$ и более, согласно [17], трубная сталь К60 сохраняет упругие свойства независимо от параметров температуры и давления и соответствует правилам [10];

- $250 D$ и менее, недопустимы по правилам [10,11];

- 250-500D требует индивидуального расчета в каждом конкретном случае на соответствие правилам [10].
Из рис. 4 видно, что рассматриваемый участок газопровода представляет собой не прямые участки с радиусом изгиба, близким к бесконечности, по проектной документации, а систему разнонаправленных радиусов.

Ненормативный радиус изгиба $282 D$ (123300 м, 6,3 часа) можно объяснить воздействием ГДЗ при направлении действия вниз вектора распределенных сил. Минимальный радиус образовался в 20 м от центра ГДЗ, где находится дно ручья (2 ОХГ на координате 123350 м). Измерения напряжений, представленных в табл. 1, были проведены в стороне от максимальных напряжений. Кроме того, можно отметить, что все ОХГ имеют радиусы пластического изгиба более проектного, равного 60 м (от 86 до 115 м), т. е. «распрямились».

В целом на данном участке протяженностью $600 \mathrm{м}$, около $60 \%$ не соответствуют требованиям правил [11] по радиусам упругого изгиба $1000 D$ и более. Аналогичные данные приводятся в работе [17], где показано, что на прямолинейном участке протяженностью 400 м имеются 11 участков с радиусом изгиба менее $1000 D$, общей протяженностью около $30 \%$. Появление технологии измерения радиусов изгиба [13] позволило объективно отражать фактическое линейно-высотное положение участка газопровода, его радиусы изгиба, их направление, что значительно расширяет возможности ВТД уже и в части оценки напряжений.

Для приведения газопровода, расположенного в зоне действия геодинамических факторов, к требованиям правил [11] необходима его практически $100 \%$ переукладка, что не представляется возможным. Более реальная альтернатива - пересмотреть существующие нормативные документы по эксплуатации МГ [18], учитывающие реальное НДС. Например, в [17] показано, что для МГ из 


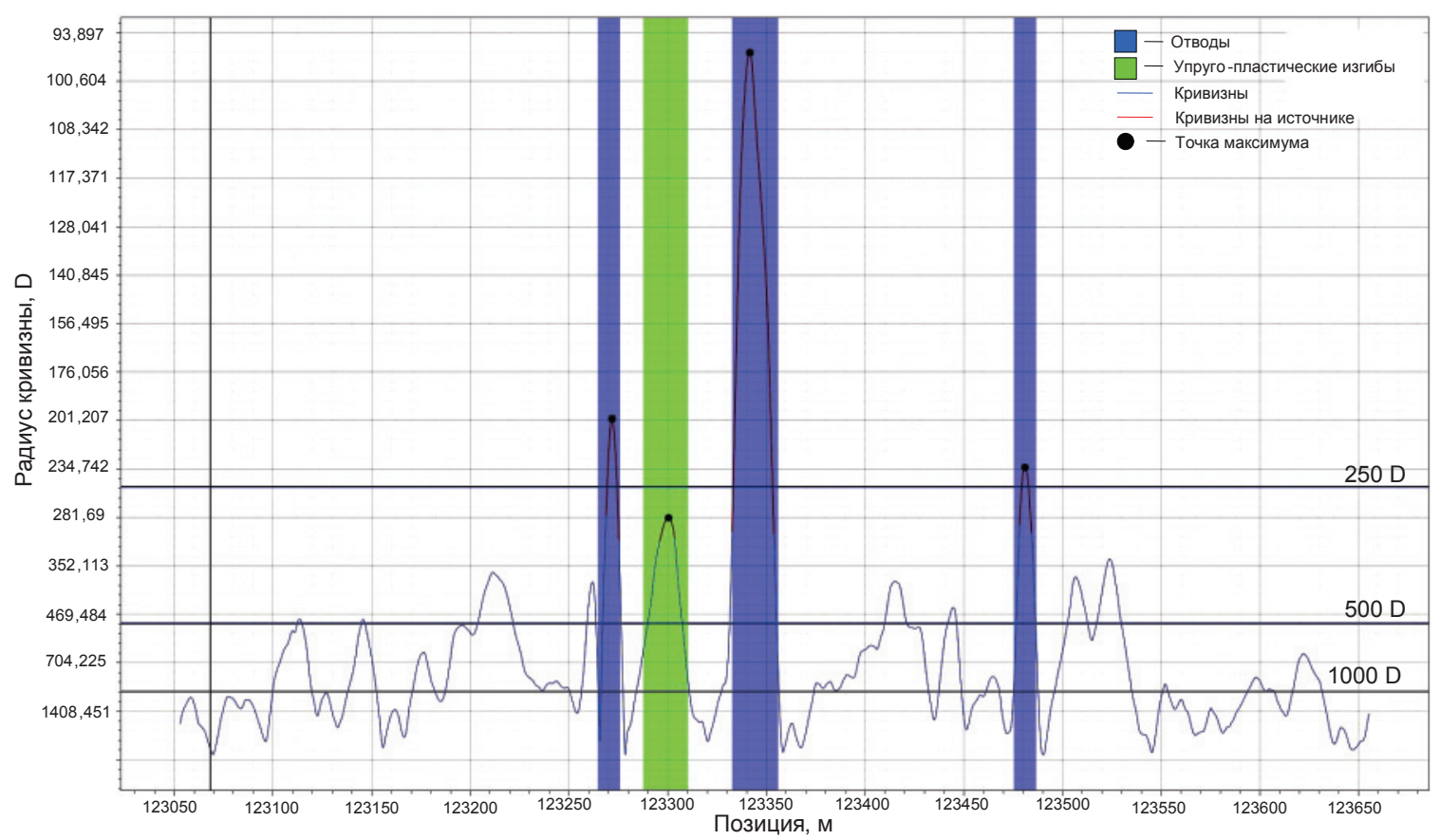

Pис. 4. Радиусы кривизны газопровода на пересечении с ГДЗ № 12

Fig. 4. Radii of pipeline curvature at the intersection with geodynamic zones 12

трубной стали К60 со сроком эксплуатации 33 лет и более, при радиусе изгиба $500 \mathrm{D}$, продольные напряжения не превышают предел пропорциональности, составляющий около $0,7 \sigma_{\text {тек }}$, что, в свою очередь, соответствует требованиям правил [10].

\section{Динамика изменения потенциально-опасных участков под воздействием активных геодинамических зон}

Динамику напряжений от изгиба газопровода можно отслеживать по данным нескольких пропусков внутритрубных дефектоскопов, а по характеру изменения радиусов кривизны также возможно выявление активных ГДЗ. В табл. 2 приводятся ПОУ с ненормативными радиусами изгиба $(500 D$ и менее), выявленные на МГ, диаметром 1420 мм, в процессе двух обследований средствами ВТД на пересечениях с ГДЗ (№ 1-12), после 33 лет (2015 г.) [16] и 35 лет (2017 г.) [19] эксплуатации.

Параметры транспорта газа (температура и давление), при пропусках снарядов, согласно отчетам ВТД [16, 19], на координате 123500 м (ГДЗ № 12) практически одинаковы:

- давление +6,2 МПа (2015), 6,7 МПа (2017);

- температура газа $+13,1^{\circ} \mathrm{C}(2015), 12,8^{\circ} \mathrm{C}(2017)$.

Температура газа при повторном пропуске на $0,3^{\circ}$ ниже, т. е. в пределах погрешности измерения, что не должно влиять на напряжения от температурного перепада.

Анализ данных табл. 2 показывает:

- в 2015 г. выявлены ПОУ на 8 ГДЗ, в 2017 г. уже на 11 ГдЗ;

- в 2015 г. было выявлено 17 ПОУ с радиусом изгиба менее 500 D, в 2017 г. было выявлено уже
50, т. е. произошло трехкратное увеличение ПОУ, что свидетельствует о повышении активности процессов ГдЗ.

Таблица 2. ПОУ на МГ на пересечениях с ГДЗ, выявленных ВТД, в 2015 и 2017 г2.

Table 2. Potentially dangerous sections on the main gas pipeline at the intersections with geodynamic zones identified by in-line diagnostics, in 2015 and 2017

\begin{tabular}{|c|c|c|c|}
\hline & $\begin{array}{c}\text { Границы ГдЗ, } \\
\text { M } \\
\text { № участка } \\
\text { Site no. } \\
\text { Borders } \\
\text { ofeodynamic } \\
\text { zones, } m\end{array}$ & $\begin{array}{c}\text { Количество ПОУ на участке } \\
\text { Number of potentially dangerous } \\
\text { sections on the site }\end{array}$ \\
\cline { 4 - 5 } & $\begin{array}{c}\text { ВТД-2015 } \\
\text { in-line diagnostics } \\
2015\end{array}$ & $\begin{array}{c}\text { BТД-2017 } \\
\text { in-line diagnostics } \\
2017\end{array}$ \\
\hline 1 & $142200-142900$ & 2 & 5 \\
\hline 2 & $140000-140900$ & 3 & 7 \\
\hline 3 & $139300-139600$ & - & 2 \\
\hline 4 & $138300-138900$ & - & 2 \\
\hline 5 & $136600-137300$ & 2 & 3 \\
\hline 6 & $135400-136000$ & 1 & 5 \\
\hline 7 & $132400-133100$ & - & 7 \\
\hline 8 & $130500-131200$ & 1 & 7 \\
\hline 9 & $128200-128700$ & 3 & 4 \\
\hline 10 & $125200-126100$ & 1 & 6 \\
\hline 12 & $123200-123600$ & 4 & 2 \\
\hline & Bсего/Total & 17 & 50 \\
\hline
\end{tabular}

Примечание. Технические параметры внутритрубных снарядов в 2015 и 2017 г2. [16, 19] не изменились.

Note. Engineering papmeters of pig recaivers in 2015 and 2017 [16, 19] have not changed.

Трубопровод, в данном случае диаметром 1420 мм, является достаточно жесткой системой, 


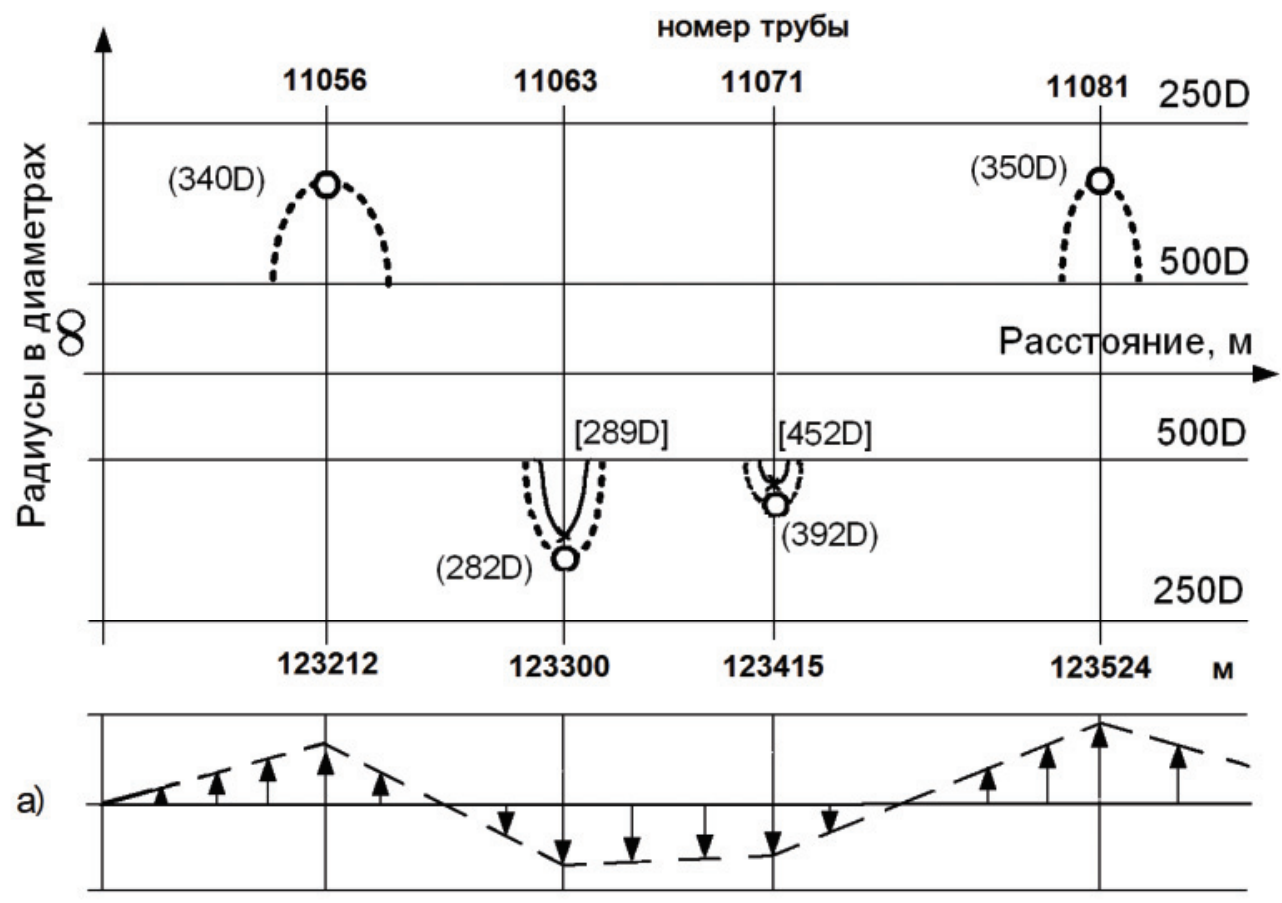

схема вектора сил до 2015 г.

๑)

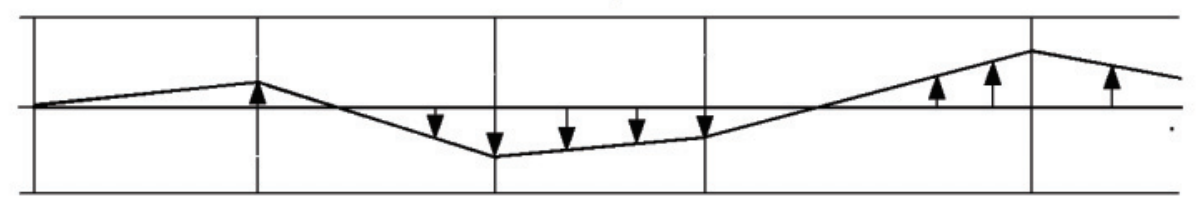

схема вектора сил с 2015 по 2017 г.

$$
\text { -.--.-- } 2015 \text { год; ——- } 2017 \text { год }
$$

Рис. 5. Радиус изгиба в ГдЗ № 12. Схема воздействия вектора распределенных сил: а) 2015 г.; б) 2017 г.

Fig. 5. Bending radius in geodynamic zone № 12. Diagram of the distributed force vector effect: a) 2015; b) 2017

которая сопротивляется линейным и радиальным перемещениям, а между защемленными участками представляет собой непрерывную натянутую нить. Для возникновения на подземном МГ ПОУ с ненормативными радиусами изгиба необходимо масштабное воздействие распределенных сил, такое может обеспечить ГДЗ. Можно предположить, что уже до второго ВТД имелись радиусы изгиба, но более $500 \mathrm{D}$.

На рис. 5 представлен график радиусов изгиба, пересекающих ГДЗ № 12, в виде укрупненной схемы, отражающий радиусы менее 500D (укрупненный, в данном случае, означает без учета радиусов на участках, где они менее $500 D$ ). На рис. 5 отражены данные двух ВТД (2015 и 2017 гг.). В верхней части схемы указаны участки, расположенные выпуклостью вверх - с 9 до 3 часов, в нижней - выпуклостью вниз - с 3 до 9 часов. На рис. 5, а схема вектора распределенных сил и профиль оси трубопровода по данным 2015 г., 5, б - по данным 2017 г.

При анализе картины НДС мы исходим из того, что газопровод построен по правилам [10, 11], т. е. на участке радиусы упругого изгиба не менее
$1000 D$, а значит выявленные несоответствия вызваны факторами, возникшими при эксплуатации.

Данные ВТД, представленные на рис. 5, совпадают с картиной, изображенной на рис. 2. То есть, центр выпуклой зоны опускается, а края поднимаются - оба ПОУ по центру выпуклостей. Таким образом с достаточной точностью определены параметры ГДЗ: направление движения блоков вертикальное протяженность 250-300 м [18].

Рассмотрим информацию, представленную на рис. 6 , для более сложного случая геодинамического воздействия на примере ГДЗ № 2 общей протяженностью $900 \mathrm{~m}$.

По данным 2015 г. (рис. $6, a)$, на участке слева, длиной около 450 м, к 33 году эксплуатации ось трубопровода направлена выпуклостью вверх [16], труба № 12578 ( $\approx \approx 360$ МПа, 11,7 часа), № 12591 (365 МПа, 11,6), 12598 (350 МПа, 11,1).

По данным 2017 г., картина НДС кардинально изменилась (рис. 6, б) - участок слева направо:

- около 300 м прогнулся вниз, напряжения [19], труба № 12562 ( $\approx \approx 306$ МПа, 6,2 часа) № 12568 (247 МПа, 6,1); 


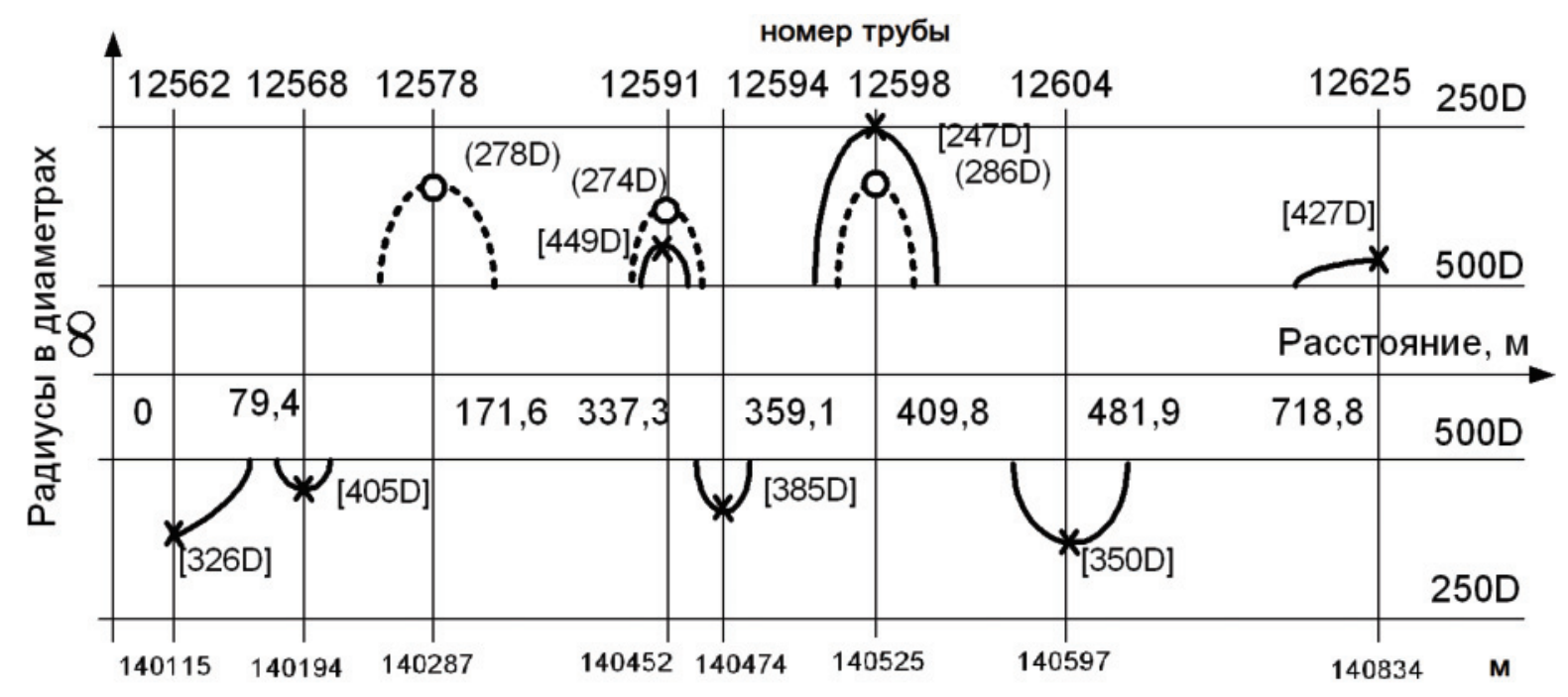

a)

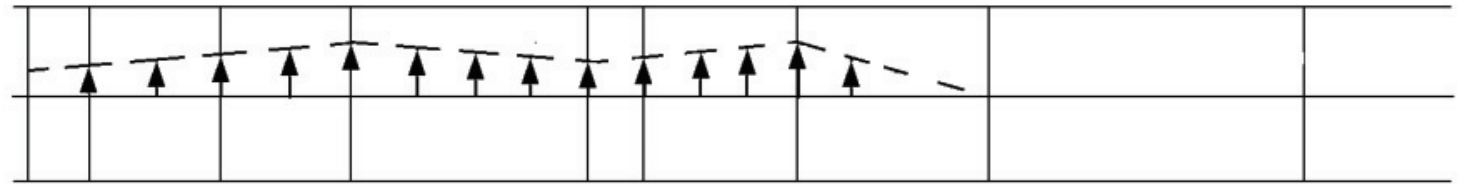

схема вектора сил до 2015 г.

6)

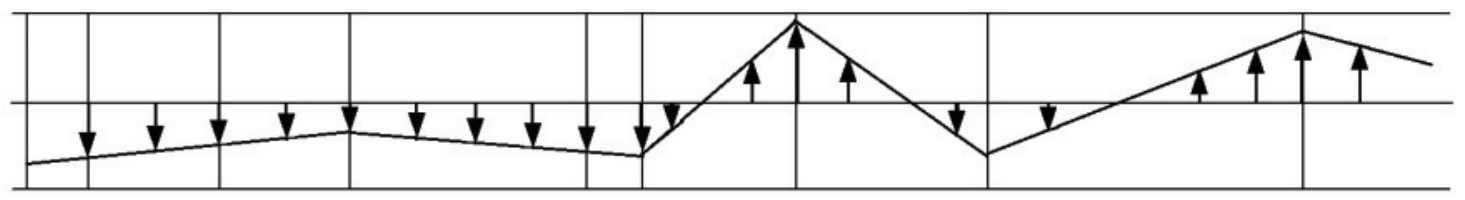

схема вектора сил с 2015 по 2017 г.

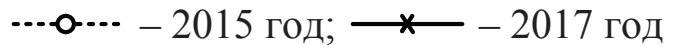

Puс.6. Величины радиусов изгиба на ГдЗ № 2. Схема воздействия вектора распределенных сил: а) до 2015 г.; б) 2015-2017 г2.

Fig. 6. Bending radius in geodynamic zone № 2. Diagram of the distributed force vector effect: a) until 2015; b) 2015-2017

- около 50 м ось выпуклая вверх, № 12591 (223 МПа, 11,4);

- около 70 м выпуклая вниз, № 12594 (260 МПа, $4,9)$;

- около 100 м выпуклая вверх, № 12598 (405 МПа, 11,9);

- около 300 м выпуклая вниз, № 12604 (286 МПа, 5,4);

- от 600 м выпуклая вверх, № 12625 (234 МПа, $11,8)$.

Ось трубопровода представляет собой полные три волны синусоиды, т. е. меняется знак изгибных напряжений и в широких пределах сами изгибные напряжения. C достаточной точностью определены границы и направление движения блоков ГДЗ средствами ВТД [18].

Кроме того, например, по трубе № 12598 прослеживается тенденция повышения изгибных напряжений с 360 МПа (2015 г.) до $405 \mathrm{MПа}$ (2017 г.), что может служить основанием для проведения ремонтно-восстановительных работ.

Из приведенных данных видно, что на участках МГ под воздействием ГДЗ за два неполных го- да между 2015 и 2017 гг. изменяются радиусы изгиба, а значит и напряжения от изгиба, что подтверждает данные РАН о современных проявлениях надвигов на западном Урале.

Кроме того, появляется возможность решения обратной задачи, с использованием ВТД определять активные ГДЗ, с определением границ и направления движения блоков [20].

На основе проведенных исследований можно сформулировать следующие выводы.

1. Приводится характеристика основных этапов общепринятой технологии выявления ГДЗ, определения их основных параметров, оценки НДС МГ включающей: аэрокосмические и геолого-геофизические технологии; измерения напряжений в трассовых условиях. Показано, что эта многоэтапная технология из разных областей науки по своим результатам является вероятностной и не позволяет достоверно выявлять ПОУ с оценкой их НДС.

2. Прорывом в области диагностики трубопроводов является технология построения профиля и определения направления и величины радиуса 
изгиба трубопровода средствами ВТД. Измерение позволяет выявлять ПОУ МГ и с достаточной для инженерных расчетов точностью оценивать напряжения от изгиба газопровода.

3. Показано, что после длительной эксплуатации (33 года и более) линейная часть МГ не соответствует требованиям правил по минимально допустимому радиусу изгиба 1000 D. B связи с невозможностью полной переукладки линейной

\section{СПИСОК ЛИТЕРАТУРЫ}

1. Late Cenozoic magmatic inflation, crustal thickening, and $>2 \mathrm{~km}$ of surface uplift in central Tibet / J.L. Chen, A. Yin, J.F. Xu, Y.H. Dong, Z.Q. Kang // Geology. - 2018. - V. 46. - P. 19-22.

2. Haproff P.J., Zuza A.V., Yin A. West-directed thrusting south of the eastern Himalayan syntaxis indicates clockwise crustal flow at the indenter corner during the India-Asia collision // Tectonophysics. -2018 . - V. 722. - P. 277-285.

3. 3D geodynamic models for the development of opposing continental subduction zones: the Hindu Kush-Pamir example / J. Liao, T. Gerya, M. Thielmann, A.A.G. Webb, S.-K. Kufner, A. Yin // Earth and Planetary Science Letters. - 2017. - V. 480. P. 133-146.

4. Late Paleozoic and Mesozoic evolution of the Lhasa Terrane in the Xainza area of southern Tibet / S.Y. Fan, L. Ding, M.A. Murphy, M. Yao, A. Yin // Tectonophysics. - 2017. - V. 721. P. $415-434$.

5. Давлетов М.И. Исходные параметры для расшифровки геологических факторов аварий трубопроводов на территории Башкортостана // Энергоэффективность. Проблемы и решения: V Российский энергетический форум. - Уфа, 2005. - С. 232-237.

6. Давлетов М.И. Неотектонические движения по зонам разломов на трассах трубопроводов северо-запада и запада Башкирии // Энергоэффективность. Проблемы и решения: V Российский энергетический форум. - Уфа, 2005. - С. 238-241.

7. Савина А.В., Гражданкин А.И. Определение минимальных безопасных расстояний от магистральных трубопроводов до населенных пунктов, зданий и сооружений // Газотранспортные системы: настоящее и будущее: сборник докладов IV Международной научно-технической конференции и выставки GTS. М., 2011. - Ч. 1 - М.: Газпром ВНИИГАЗ, 2011. - С. 320-324.

8. Аскаров Р.М., Мазитов Д.Г., Рафиков С.К. Прогноз напряженно-деформированного состояния участков газопроводов, пересекающих геодинамические зоны // Проблемы сбора, подготовки и транспорта нефти и нефтепродуктов. - 2015. - № 1. C. $121-126$

9. Аскаров Р.М., Мазитов Д.Г., Рафиков С.К. Оценка фактических напряжений магистральных газопроводов, пересекающих геодинамические зоны // Проблемы сбора, подготовки и транспорта нефти и нефтепродуктов. - 2015. - № 2 (100). C. $136-143$. части МГ ПАО «Газпром» предлагается переработать нормативные требования по допустимым напряжениям.

4. Обследования МГ средствами ВТД позволяют при повторном пропуске оценивать динамику изменения радиусов изгиба, а значит и его НДС.

5. Показана возможность, по данным ВТД, выявления активных ГДЗ и их параметров: границ и направления движения блоков.

10. Свод правил 36.13330.2012. Магистральные трубопроводы (Актуализированная редакция СНиП 2.05.06-85* с изменениями и дополнениями). URL: http://docs.cntd.ru/document/1200103173 (дата обращения: 13.05.2018).

11. Свод правил 86.13330.2012. Магистральные трубопроводы (Актуализированная редакция СНиП III-42-80*). URL: http://docs.cntd.ru/document/1200102566 (дата обращения: 13.05.2018).

12. Чучкалов М.В. Разработка методов выявления, торможения и предотвращения коррозионного растрескивания под напряжением на магистральных газопроводах: дис. ... д-ра техн. наук. - Уфа, 2015. - 321 c.

13. Способ определения потенциально опасного участка трубопровода с непроектным уровнем напряженно-деформированного состояния: пат. № 2602327 РФ. Заявл. 04.04.2015; опубл. 20.11.2016, Бюл. № 32 .

14. Successful management of the pipeline cracking threat using an ultrasonic in-line inspection tool - a case study / B. Delanty, I. Whitbread, M. Maxwell, J. Munro // Pipelines international. 2015. - № 9. - P. 44-48.

15. Pipeline Operators Forum. Specifications and requirements for intelligent pig inspection of pipelines. Version 2016. - СПб.: ЛТД «ДИАС», 2016. $-56 \mathrm{p}$.

16. Отчет по внутритрубной дефектоскопии газопровода Уренгой-Петровск. - Екатеринбург: НПЦ Внутритрубная диагностика, 2015. - $974 \mathrm{c.}$

17. 0 фактических радиусах изгиба линейной части магистральных газопроводов / Р.М. Аскаров, К.М. Гумеров, А.Н. Кукушкин, И.М. Исламов // Трубопроводный транспорт: теория и практика. - 2017. - № 6. - С. 28-33.

18. Отчет по внутритрубной дефектоскопии газопровода Уренгой-Петровск. - Екатеринбург: НПЦ Внутритрубная диагностика, 2017. - 1032 с.

19. СТО Газпром 2-3.5-454-2010. Правила эксплуатации магистральных газопроводов. - М.: 000 «Газпром экспо», 2010. $241 \mathrm{c.}$

20. Способ выявления геодезических зон, пересекающих магистральные трубопроводы: пат. № 2666387 РФ. Заявл. 04.04.2017; Опубл. 07.09.2018, Бюл. № 25.

Поступила: 14.11.2018 2.

\section{Информация об авторах}

Аскаров P.M., доктор технических наук, доцент кафедры транспорта и хранения нефти и газа Уфимского Государственного нефтяного технического университета.

Валеев A.P., кандидат технических наук, доцент кафедры транспорта и хранения нефти и газа Уфимского Государственного нефтяного технического университета.

Ислалов И.М., начальник Ургалинского линейно-производственного управления.

Tагиров М.Б., инженер 2 категории Инженерно-технического центра 000 «Газпром трансгаз Уфа». 
UDC 622.692.4.053

\title{
ANALYSIS OF THE EFFECT OF GEODYNAMIC ZONES CROSSING THE MAIN GAS PIPELINES ON THEIR STRESS-STRAIN STATE
}

\author{
Robert M. Askarov', \\ askarov1943@mail.ru
}

Anvar R. Valeev', anv-v@yandex.ru

Ildar M. Islamov², mildarislamov@mail.ru

\author{
Marsel B. Tagirov ${ }^{3}$, \\ tagirovmarse|@gmail.com \\ 1 Ufa State Petroleum Technological University, \\ 1, Kosmonavtov street, Ufa, 450062, Russia. \\ 2 Limited Liability Company «Gazprom transgaz Ufa», \\ 59, R. Zorge street, Ufa, 450054, Russia. \\ ${ }^{3}$ Engineering and Technology Center Gazprom Transgas Ufa, \\ 11, Sipaylovskaya street, Ufa, 450099, Russia.
}

The relevance. One of the reasons capable of destroying gas pipelines is geodynamic effects, since with long-term operation of gas pipelines located, for example, on tectonic faults, the damaging factor due to bending stresses is accumulated. The analysis of the actual position of gas pipelines according to in-line technical diagnostics showed the presence of potentially dangerous areas, in which the curvature does not match the design, and therefore the bending stresses can also differ significantly in absolute magnitude in the larger direction from the design. Therefore, it is necessary to identify such potentially dangerous areas in a timely manner and, depending on the level of actual stresses, carry out restoration measures.

The main aim of research is to analyze the influence of geodynamic zones crossing the main gas pipelines on their stress-strain state using the data from in-line inspection and taking into account their operation beyond the depreciation period.

Objects: four thread corridor of the main gas pipeline running through the western Urals.

Methods: analytical methods for the study of the stress-strain state of gas pipelines according to the data on the spatial position of its axis, obtained by the methods of the generally accepted technology and the data of the in-line technical diagnostics.

Results. The authors have analyzed the main stages of the generally accepted technology for detecting geodynamic zones and determining its main parameters, the assessment of stress-strain state. The paper demonstrates the possibility of detecting potentially dangerous areas at intersections with geodynamic zones, according to the in-line technical diagnostics. It was established that with the increase of the gas pipeline service life, the number of potentially dangerous areas grows, in which the radius of curvature does not meet the requirements of regulatory documentation for the minimum allowable equal to $1000 \mathrm{D}$. Based on the results of the data processing of the in-line technical diagnostics, the examples of determining the values of bending stresses exceeding the standard values are given. As it is impossible to complete re-laying of gas pipeline, it is proposed to bring regulatory requirements for the level of stresses. In addition, the possibility of identifying active geodynamic zones and their parameters, according to the data of the in-line technical diagnostics, is shown.

Key words:

Gas pipeline, geodynamic zones, stress-strain state, potentially dangerous areas, bend radius of the pipeline, in-line diagnostics.

\section{REFERENCES}

1. Chen J.L., Yin A., Xu J.F., Dong Y.H., Kang Z.Q. Late Cenozoic magmatic inflation, crustal thickening, and $>2 \mathrm{~km}$ of surface uplift in central Tibet. Geology, 2018, vol. 46, pp. 19-22.

2. Haproff P.J., Zuza A.V., Yin A. West-directed thrusting south of the eastern Himalayan syntaxis indicates clockwise crustal flow at the indenter corner during the India-Asia collision. Tectonophysics, 2018, vol. 722, pp. 277-285.

3. Liao J., Gerya T., Thielmann M., Webb A.A.G., Kufner S.-K., Yin A. 3D geodynamic models for the development of opposing continental subduction zones: The Hindu Kush-Pamir example. Earth and Planetary Science Letters, 2017, vol. 480, pp. $133-146$.

4. Fan S.Y., Ding L., Murphy M.A., Yao M., Yin A. Late Paleozoic and Mesozoic evolution of the Lhasa Terrane in the Xainza area of southern Tibet. Tectonophysics, 2017, vol. 721, pp. 415-434
5. Davletov M.I. Iskhodnye parametry dlya rasshifrovki geologicheskikh faktorov avariy truboprovodov na territorii Bashkortostana [Initial parameters for interpreting geological factors of pipeline accidents in the territory of Bashkortostan]. $V$ Rossiyskiy energeticheskiy forum. Energoeffektivnost. Problemy i resheniya [V Russian Energy forum. Power efficiency. Problems and solutions]. Ufa, 2005. pp. 232-237.

6. Davletov M.I. Neotektonicheskie dvizheniya po zonam razlomov na trassakh truboprovodov severo-zapada i zapada Bashkirii [Neotectonic movements along the fault zones along the pipelines of the northwest and west of Bashkiria]. $V$ Rossiyskiy energeticheskiy forum. Energoeffektivnost. Problemy i resheniya [V Russian Energy forum. Power efficiency. Problems and solutions]. Ufa, 2005. pp. 238-241

7. Savina A.V., Grazhdankin A.I. Opredelenie minimalnykh bezopasnykh rasstoyaniy ot magistralnykh truboprovodov do naselen- 
nykh punktov, zdaniy i sooruzheniy [Determination of the minimum safe distances from trunk pipelines to settlements, buildings and structures]. Gazotransportnye sistemy: nastoyashchee i budushchee. Sbornik dokladov IV Mezhdunarodnoy nauchnotekhnicheskoy konferentsii $i$ vystavki GTS [Gas-transport systems: present and future. Reports of the IV International scientific and technical conference and exhibition GTS]. Moscow, 2011. P. 1. Moscow, Gazprom VNIIGAZ Publ., 2011. pp. $320-324$.

8. Askarov R.M., Mazitov D.G., Rafikov S.K. Prognoz napryazhenno-deformirovannogo sostoyaniya uchastkov gazoprovodov, peresekayushchikh geodinamicheskiye zony [The forecast of the stress-strain state of sections of gas pipelines crossing the geodynamic zones]. Problemy sbora, podgotovki i transporta nefti i nefteproduktov, 2015, no. 1, pp. 121-126.

9. Askarov R.M., Mazitov D.G., Rafikov S.K. Otsenka fakticheskikh napryazheniy magistralnykh gazoprovodov, peresekayushchikh geodinamicheskiye zony [Evaluation of the actual voltages of gas pipelines crossing geodynamic zones]. Problemy sbora, podgotovki i transporta nefti i nefteproduktov, 2015, no. 2 (100), pp. $136-143$.

10. SP 36.13330.2012 Magistralnye truboprovody (Aktualizirovannaya redaktsiya SNiP $2.05 .06-85^{*}$ s izmeneniyami i dopolneniyami) [Main pipelines (Updated edition of SNiP 2.05.06-85* with changes and additions).]. Available at: http://docs.cntd.ru/document/1200103173 (accessed 13 May 2018).

11. SP 86.13330.2012. Magistralnye truboprovody (Aktualizirovannaya redaktsiya SNiP III-42-80*) [Main pipelines (Updated edition of SNiP III-42-80* with changes and additions)]. Available at: http://docs.cntd.ru/document/1200102566 (accessed 13 May 2018).

12. Chuchkalov M.V. Razrabotka metodov vyavleniya, tormozheniya $i$ predoturashcheniya korrozionnogorastreskivaniya pod napryazheniem na magistralnykh gazoprovodakh. Dis. Dokt. nauk [development of tehniques for detecting, restraining and preventing corrosion cracking under stress on main gaslines. Dr. Diss.]. Ufa, 2015. $321 \mathrm{p}$.
13. Usmanov R.R., Chuchkalov M.V., Askarov R.M., Zakiryanov R.V. Sposob opredeleniya potentsialno opasnogo uchastka truboprovoda s neproyektnym urounem napryazhenno-deformirovannogo sostoyaniya [The method of determining the potentially dangerous section of the pipeline with a non-design level of stressstrain state]. Patent RF, no. 2602327, 2016.

14. Delanty B., Whitbread I., Maxwell M., Munro J. Successful management of the pipeline cracking threat using an ultrasonic in-line inspection tool - a case study. Pipelines international, 2015, no. 9, pp. 44-48.

15. Pipeline Operators Forum. Specifications and requirements for intelligent pig inspection of pipelines. Version 2016. St. Petersburg, LTD «DIAS Publ., 2016. 56 p.

16. Otchet po vnutritrubnoy defektoskopii gazoprovoda Urengoy-Petrousk [Report on the in-line inspection of the Urengoy-Petrovsk gas pipeline]. Moscow, NPTS Vnutritrubnaya diagnostika, 2015. $974 \mathrm{p}$.

17. Askarov R.M., Gumerov K.M., Kukushkin A.N., Islamov I.M. 0 fakticheskikh radiusakh izgiba lineynoy chasti magistralnykh gazoprovodov [0 $\mathrm{n}$ actual bending radius of the linear part of gas pipelines]. Truboprovodny transport: teoriya i praktika, 2017, no. 6, pp. 28-33.

18. Otchet po unutritrubnoy defektoskopii gazoprovoda Urengoy-Petrousk [Report on the in-line inspection of the Urengoy-Petrovsk gas pipeline]. Moscow, NPTS Vnutritrubnaya diagnostika, 2017. $1032 \mathrm{p}$.

19. STO Gazprom 2-3.5-454-2010. Pravila ekspluatatsii magistralnykh gazoprovodov [Rules for the operation of gas pipelines.]. Moscow, Gazprom, 2010. 241 p.

20. Mastobaev B.N., Askarov R.M., Rafikov S.K., Kitaev S.V., Mazitov D.G., Chuchkalov M.V., Kukushkin A.N. Sposob vyyavleniya geodezicheskikh zon, peresekayushchikh magistralnyye truboprovody [Method for identifying geodesic zones crossing main pipelines]. Patent RF, no. 2666387, 2018.

Received: 14 November 2018.

\section{Information about the authors}

Robert M. Askarov, Dr. Sc., associate professor, Ufa State Petroleum Technological University.

Anvar R. Valeev, Cand. Sc., associate professor, Ufa State Petroleum Technological University.

Ildar M. Islamov, head of Urgalinskoe LPA «Gazprom transgaz Ufa» LLC.

Marsel B. Tagirov, Engineer of the 2 category, Engineering and Technology Center Gazprom Transgas Ufa. 